\title{
Heat Transfer Analysis of MHD Water Functionalized Carbon Nanotube Flow over a Static/Moving Wedge
}

\author{
Waqar A. Khan, ${ }^{1}$ Richard Culham, ${ }^{1}$ and Rizwan Ul Haq ${ }^{2}$ \\ ${ }^{1}$ Department of Mechanical and Mechatronics Engineering, University of Waterloo, Waterloo, ON, Canada N2L 3G1 \\ ${ }^{2}$ Department of Mathematics, Quaid-i-Azam University, Islamabad 45320, Pakistan \\ Correspondence should be addressed to Waqar A. Khan; wkhan_2000@yahoo.com
}

Received 6 November 2014; Accepted 2 March 2015

Academic Editor: Theodorian Borca-Tasciuc

Copyright (c) 2015 Waqar A. Khan et al. This is an open access article distributed under the Creative Commons Attribution License, which permits unrestricted use, distribution, and reproduction in any medium, provided the original work is properly cited.

\begin{abstract}
The MHD flow and heat transfer from water functionalized CNTs over a static/moving wedge are studied numerically. Thermal conductivity and viscosity of both single and multiple wall carbon nanotubes (CNTs) within a base fluid (water) of similar volume are investigated to determine the impact of these properties on thermofluid performance. The governing partial differential equations are converted into nonlinear, ordinary, and coupled differential equations and are solved using an implicit finite difference method with quasi-linearization techniques. The effects of volume fraction of CNTs and magnetic and wedge parameters are investigated and presented graphically. The numerical results are compared with the published data and are found to be in good agreement. It is shown that the magnetic field reduces boundary layer thickness and increases skin friction and Nusselt numbers. Due to higher density and thermal conductivity, SWCNTs offer higher skin friction and Nusselt numbers.
\end{abstract}

\section{Introduction}

Many manufacturing procedures consist of continuous stretching surfaces cooled by an external stream along the production line. Boundary layer flow behavior over a moving continuous solid surface is a significant type of flow happening in several industrial processes. The examples are the thermal processing of sheet-like materials which is a necessary operation in the production of paper, linoleum, polymeric sheets, wire drawing, drawing of plastic films, metal spinning, roofing shingles, insulating materials, finefiber matts, cooling of films or sheets, conveyor belts, metallic plates, and cylinders. In virtually all such processing operations, the sheet moves parallel to its own plane. The moving sheet may induce motion in the neighboring fluid or, alternatively, the fluid may have an independent forced convection motion that is parallel to that of the sheet. Both the kinematics of stretching and the simultaneous heating or cooling during such processes have a decisive influence on the quality of the final products. In virtually all such processing operations, the sheet moves parallel to its own plane.
The pioneering works of Falkner and Skan [1], Hartree [2], and Koh and Hartnett [3] provided the fundamental solutions for wedge problems and extended wedge problem in different directions. They solved governing equations for laminar flow of conventional fluids over permeable wedges with suction, including isothermal and variable wall temperature distributions, and calculated the heat transfer and skin friction for laminar flow. Takhar and Pop [4] studied the problem of MHD heat transfer from a wedge with a uniform surface temperature in a fluid of large Prandtl numbers. They obtained a closed form solution and compared their results with published data. H.-T. Lin and L.-K. Lin [5] proposed a similarity solution method to determine accurate solutions for laminar forced convection heat transfer from both isothermal and uniform-flux wedges to fluids of any Prandtl number. They presented a simple correlation equation for any wedge and any Prandtl number. Watanabe [6] investigated theoretically the behavior of forced flow over a wedge with uniform suction or injection. The resulting integral equations were solved by iterative numerical quadratures. Yih [7] investigated the effects of viscous dissipation and stress work 
on the MHD forced convection adjacent to a nonisothermal wedge. They used the Keller box method and presented the numerical results for the local friction coefficient and the local Nusselt number.

Hossain et al. [8] considered forced flow and heat transfer of a viscous incompressible fluid having temperaturedependent viscosity and thermal conductivity past a wedge with a uniform surface heat flux. They presented the local skin friction coefficient and rate of heat transfer for various values of the governing parameters. Pal and Mondal [9] analyzed heat transfer characteristics of an incompressible Newtonian electrically conducting and heat generating/absorbing fluid having temperature-dependent viscosity over a nonisothermal wedge in the presence of thermal radiation. They included the effects of viscous dissipation, Joule heating, stress work, and thermal radiation and obtained numerical results for the velocity and temperature profiles as well as the local skin friction coefficient and local Nusselt number. Ishak et al. [10] investigated MHD boundary layer flow past a wedge with constant surface heat flux immersed in an incompressible micropolar fluid. They employed the Keller box method to obtain numerical results and showed that micropolar fluids display drag reduction and consequently reduce the heat transfer rate at the surface. Su et al. [11] proposed a new analytical method named DTM-BF and applied their study to MHD mixed convective flow and heat transfer of an electrically conducting fluid over a stretching permeable wedge in the presence of thermal radiation and Ohmic heating. They presented the effects of various embedded parameters and dimensionless parameters on the skin friction and heat transfer. Prasad et al. [12] obtained numerical solutions for the hydromagnetic mixed convection boundary layer flow of an electrically conducting fluid over a nonisothermal wedge. They investigated various effects of the embedded dimensionless parameters. Rahman et al. [13] discovered the effects of thermophoresis on an unsteady MHD forced convective heat and mass transfer flow of an electrically conducting fluid over a wedge with variable electrical conductivity. They solved locally similar ordinary differential equations by applying Nachtsheim-Swigert shooting iteration technique along with a sixth order Runge-Kutta integration scheme. They found that the skin friction coefficient, Nusselt number, and Sherwood number are higher for the fluids of constant electrical conductivity than those of the variable electrical conductivity. Muhaimin et al. [14] analyzed the effects of thermophoresis particle deposition and temperature-dependent viscosity on unsteady MHD mixed convective heat and mass transfer of an electrically conducting fluid past a porous wedge in the presence of a chemical reaction. They compared their results with those known from the literature and found excellent agreement. They found that the flow field is influenced appreciably by the applied magnetic field and thermophoresis particle deposition. Abbasbandy and Hayat [15] determined the solution of the Falkner-Skan flow by using a Homotopy Analysis method. They obtained the skin friction coefficient and compared their results with published data. Fang and Zhang [16] obtained an exact analytical solution of the famous Falkner-Skan equation which involves the boundary layer flow over a moving wall with mass transfer in the presence of a free stream. They analyzed the effects of mass transfer and wall stretching.

It is well known that convectional heat transfer fluids like water or oil are poor heat transfer fluids due to lower thermal conductivity. To enhance heat transfer, thermal conductivity of these fluids is increased by adding nano/microsized particles of higher thermal conductivity. These fluids are known as nanofluids. Following the introduction of this idea by Choi [17], several researchers including [18-23] used nanofluids to improve heat transfer from different geometries under different thermal boundary conditions. Khan and Pop [24] studied flow and heat transfer from a moving wedge in a water-based nanofluid. They investigated the effects of the governing parameters on the dimensionless velocity, temperature, and nanoparticle volume fraction and presented their results graphically. Yacob et al. [25] studied the problem of the steady flow and heat transfer over a static or moving wedge with a prescribed surface heat flux in a nanofluid. They analyzed and discussed three different types of nanoparticles with water as the base fluid. They found that the skin friction coefficient and the heat transfer rate are highest for a copperwater nanofluid compared to the other nanofluids considered in their study. Rahman et al. [26] investigated numerically the heat transfer characteristics of water-based nanofluids over a wedge with slip and a convective surface. They showed that the nanofluid velocity is lower than the velocity of the base fluid and the existence of the nanofluid leads to the thinning of the hydrodynamic boundary layer. They also found that the rate of heat transfer increases with an increase of the surface convection and the slip parameters. Later on, Rahman et al. [27] extended their study to include thermal radiation and showed that the rate of heat transfer in a nanofluid in the presence of thermal radiation significantly depends on the surface convection parameter. Chamkha et al. [28] studied mixed convection boundary layer flow over an isothermal vertical wedge embedded in a porous medium saturated with a nanofluid. They included thermal radiation and nanofluid parameters in the analysis. They presented numerical results for the velocity, temperature, and volume fraction, the local Nusselt and Sherwood numbers. Kameswaran et al. [29] presented a mathematical model for the two-dimensional steady incompressible convective heat and mass transfer from an isothermal wedge immersed in a nanofluid. They considered two types of water-based nanofluids and included the effects of the volume fraction parameter.

While there are many materials that can be used to form nanoparticles, carbon is very promising due to its high thermal, electrical, and mechanical properties [30]. A carbon nanotube is composed of a single wall (SWCNT) or multiwall (MWCNT). The thermal properties of carbon nanotubes CNT suspensions are found to be much higher than those of other nanoparticles with the same volume fraction [31-33]. They noticed enhancement in thermal properties of nanofluids with temperature and volume fraction. Ding et al. [34] investigated the heat transfer behavior of aqueous suspensions of multiwalled carbon nanotubes (CNT nanofluids) flowing through a horizontal tube. They observed significant enhancement of the convective heat transfer. Later on, Halelfadl et al. [35] extended these findings to include 


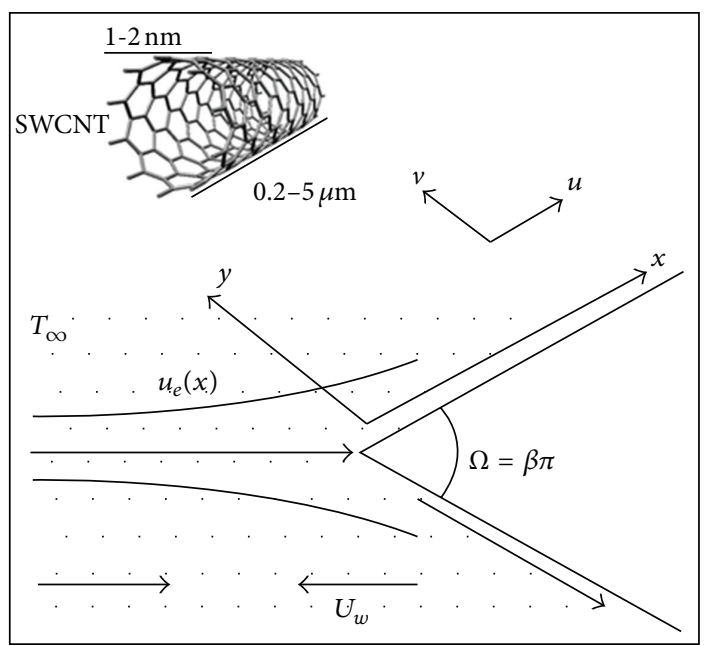

(a)

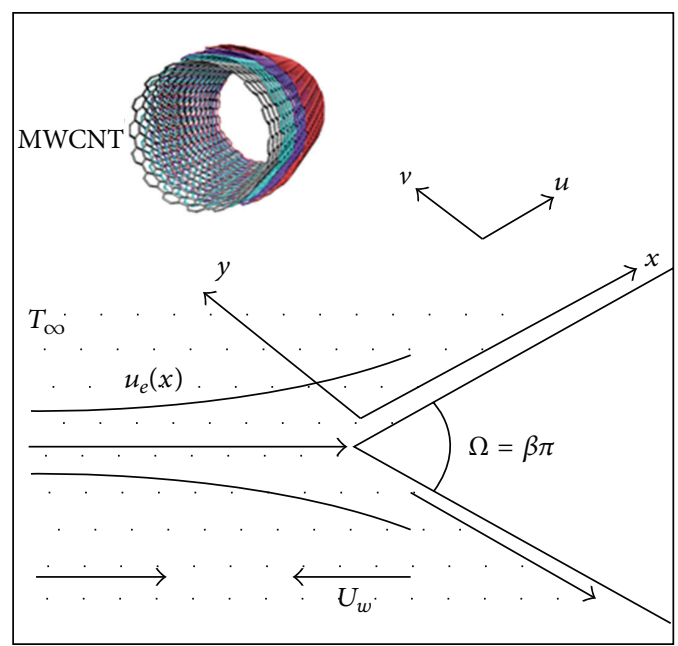

(b)

FIgURE 1: Geometry of the problem.

a coaxial heat exchanger under a laminar flow regime within the range of Reynolds numbers 500-2500 and measured thermal and rheological properties of the nanofluids. They studied the effects of the aspect ratio of carbon nanotubes, the type of base fluid and surfactant on viscosity, thermal conductivity, and laminar convective heat transfer. Khan et al. [36] employed homogeneous flow model to study the flow and heat transfer of carbon nanotubes (CNTs) along a flat plate subjected to Navier slip and uniform heat flux boundary conditions. They investigated the effects of the governing parameters on the dimensionless velocity, temperature, skin friction, and Nusselt numbers and presented numerical results in graphical and tabular forms. Recently Ul Haq et al. [37] studied thermophysical effects of carbon nanotubes on MHD flow over a stretching sheet. They considered three types of base fluids specifically water, ethylene glycol, and engine oil with single and multiwalled carbon nanotubes and found that the engine oil provides the highest skin friction and heat transfer rates.

The above literature review reveals that there are no studies that investigate thermal performance of carbon nanotubes over static or moving wedges. The objective of this study is to explore the heat transfer properties of water-based nanofluids containing SWCNTs and MWCNTs. To the best of our knowledge, the results of this paper are original and they have not been published before.

\section{Mathematical Model}

Consider steady two-dimensional boundary layer flow past a static or a moving wedge in a water-based nanofluid containing SWCNTs and MWCNTs. The physical model and geometrical coordinates are depicted in Figure 1.

The nanofluid is assumed incompressible and the flow is assumed to be steady and laminar. It is also assumed that the base fluid (i.e., water) and the nanoparticles are in thermal equilibrium and no slip occurs between them. The
TABLE 1: Thermophysical properties of water and CNTs $[41,42]$.

\begin{tabular}{lccc}
\hline $\begin{array}{l}\text { Thermophysical } \\
\text { properties }\end{array}$ & $\begin{array}{c}\text { Base fluid } \\
\text { Water }\end{array}$ & \multicolumn{2}{c}{ Carbon nanotubes } \\
& 997 & 2600 & MWCNT \\
\hline$\rho\left(\mathrm{kg} / \mathrm{m}^{3}\right)$ & 4179 & 425 & 7900 \\
$c_{p}(\mathrm{~J} / \mathrm{kg}-\mathrm{K})$ & 0.613 & 6600 & 3000 \\
$k(\mathrm{~W} / \mathrm{m}-\mathrm{K})$ & 6.2 & - & - \\
$\operatorname{Pr}$ & & & \\
\hline
\end{tabular}

thermophysical properties of the fluid and nanoparticles are given in Table 1 . Further, it is assumed that the velocity of the free stream (inviscid flow) is $u_{e}(x)=U_{\infty} x^{m}$ and that of the moving wedge is $u_{w}(x)=U_{w} x^{m}$, where $U_{\infty} ; U_{w}$ and $m$ are constants with $0 \leq m \leq 1$. We consider a two-dimensional Cartesian coordinate system $(x, y)$, where $x$ and $y$ are the coordinates measured along the surface of the wedge and normal to it, respectively. Under the boundary layer approximations, the basic steady conservation of mass, momentum, and energy equations can be written as

$$
\begin{gathered}
\frac{\partial u}{\partial x}+\frac{\partial v}{\partial y}=0 \\
u \frac{\partial u}{\partial x}+v \frac{\partial u}{\partial y}=U_{e} \frac{d U_{e}}{d x}+v_{\mathrm{nf}} \frac{\partial^{2} u}{\partial^{2} y}+\frac{\sigma B_{o}^{2}}{\rho_{\mathrm{nf}}}\left(U_{e}-u\right) \\
u \frac{\partial T}{\partial x}+v \frac{\partial T}{\partial y}=\alpha_{\mathrm{nf}} \frac{\partial^{2} T}{\partial z^{2}}+\frac{\nu_{\mathrm{nf}}}{\left(\rho c_{p}\right)_{\mathrm{nf}}}\left(\frac{\partial u}{\partial y}\right)^{2}
\end{gathered}
$$

Subject to the boundary conditions

(1) static wedge

$$
\begin{aligned}
& v=0, \quad u=0, \quad T=T_{w} \quad \text { at } y=0, \\
& u=u_{e}(x), \quad T=T_{w} \quad \text { as } y \longrightarrow \infty
\end{aligned}
$$


(2) moving wedge

$$
\begin{gathered}
v=0, \quad u=u_{w}(x), \quad T=T_{w} \quad \text { at } y=0, \\
u=u_{e}(x), \quad T=T_{w} \quad \text { as } y \longrightarrow \infty .
\end{gathered}
$$

Here $u$ and $v$ are the velocity components along the $x$ - and $y$ axes, respectively. $T$ is the temperature of the base fluid, $v_{n f}$ is viscosity of the nanofluid, $\rho_{\mathrm{nf}}$ is the density of the nanofluid, and $\alpha_{\mathrm{nf}}$ is the thermal diffusivity of nanofluid, which are given by

$$
\begin{gathered}
\nu_{\mathrm{nf}}=\frac{\mu_{\mathrm{nf}}}{\rho_{\mathrm{nf}}}, \quad \mu_{\mathrm{nf}}=\frac{\mu_{f}}{(1-\phi)^{2.5}}, \\
\alpha_{\mathrm{nf}}=\frac{k_{\mathrm{nf}}}{\rho_{\mathrm{nf}}\left(C_{p}\right)_{\mathrm{nf}}}, \quad \rho_{\mathrm{nf}}=(1-\phi) \rho_{f}+\phi \rho_{\mathrm{CNT}}, \\
\left(\rho C_{p}\right)_{\mathrm{nf}}=(1-\phi)\left(\rho C_{p}\right)_{f}+\phi\left(\rho C_{p}\right)_{\mathrm{CNT}}, \\
\frac{k_{\mathrm{nf}}}{k_{f}}=\left(1-\phi+2 \phi \frac{k_{\mathrm{CNT}}}{k_{\mathrm{CNT}}-k_{f}} \ln \frac{k_{\mathrm{CNT}}+k_{f}}{2 k_{f}}\right) \\
\cdot\left(1-\phi+2 \phi \frac{k_{f}}{k_{\mathrm{CNT}}-k_{f}} \ln \frac{k_{\mathrm{CNT}}+k_{f}}{2 k_{f}}\right)^{-1},
\end{gathered}
$$

where $\mu_{f}$ is the viscosity of base fluid, $\phi$ is the nanoparticle fraction, $\left(\rho C_{p}\right)_{\mathrm{nf}}$ is the effective heat capacity of CNTs, $k_{\mathrm{nf}}$ is the thermal conductivity of nanofluid, $k_{f}$ and $k_{\mathrm{CNT}}$ are the thermal conductivities of the base fluid and CNTs, respectively, and $\rho_{f}$ and $\rho_{\mathrm{CNT}}$ are the thermal conductivities of the base fluid and CNTs, respectively. Introducing the following similarity transformations,

$$
\begin{gathered}
\psi=\left[\frac{2 v_{f} x u_{e}(x)}{m+1}\right]^{1 / 2} f(\eta), \\
\theta(\eta)=\frac{T-T_{\infty}}{T_{w}-T_{\infty}}, \\
\eta=\left[\frac{(m+1) u_{e}(x)}{2 v_{f} x}\right]^{1 / 2} y,
\end{gathered}
$$

where $\psi$ is the stream function and is defined in the usual way as $u=\partial \psi / \partial y$ and $v=-\partial \psi / \partial x$ so as to identically satisfy (1) and $v_{f}$ is the kinematic viscosity of the base fluid. On substituting (7) into (2) and (3) along with the boundary conditions defined in (4) and (5), we obtain the following ordinary differential equations:

$$
\begin{aligned}
& \frac{1}{(1-\phi)^{2.5}\left(1-\phi+\phi \rho_{\mathrm{CNT}} / \rho_{f}\right)} f^{\prime \prime \prime} \\
& +f f^{\prime \prime}+\left(\frac{2 m}{m+1}\right)\left(1-f^{\prime 2}\right) \\
& +\frac{M^{2}}{\left(1-\phi+\phi \rho_{\mathrm{CNT}} / \rho_{f}\right)}\left(1-f^{\prime 2}\right)=0,
\end{aligned}
$$

$$
\begin{aligned}
& \frac{1}{\operatorname{Pr}} \frac{k_{\mathrm{nf}} / k_{f}}{\left\{1-\phi+\phi\left(\rho c_{p}\right)_{\mathrm{CNT}} /\left(\rho c_{p}\right)_{f}\right\}} \theta^{\prime \prime} \\
& \quad+f \theta^{\prime}+\frac{E c}{(1-\phi)^{2.5}} f^{\prime \prime 2}=0,
\end{aligned}
$$

subject to the boundary conditions

$$
\begin{gathered}
f(0)=0, \quad f^{\prime}(0)=\lambda, \quad \theta(0)=1 \\
f^{\prime} \longrightarrow 1, \quad \theta \longrightarrow 0, \quad \text { as } \eta \longrightarrow \infty,
\end{gathered}
$$

where the primes denote differentiation with respect to $\eta$. The parameters $\lambda, \beta$, and Pr are defined as

$$
\lambda=\frac{U_{w}}{U_{\infty}}, \quad \beta=\frac{2 m}{m+1}, \quad \operatorname{Pr}=\frac{\nu_{f}}{\alpha_{f}},
$$

where $\lambda$ is the constant moving wedge parameter with $\lambda>$ 0 and $\lambda<0$ correspond to a moving wedge in the same and opposite directions to the free stream, respectively, while $\lambda=0$ corresponds to a static wedge. Further, $\beta$ is the Hartree pressure gradient parameter which corresponds to $\beta=\Omega / \pi$ for a total wedge angle $\Omega$. According to White [38], positive value of $\beta$ means the pressure gradient is negative or favorable and negative values of $\beta$ denote an unfavorable pressure gradient. Further, $\beta=0(m=0)$ represents the boundary layer flow past a horizontal flat plate, while $\beta=1(m=1)$ corresponds to the boundary layer flow near the stagnation point of a vertical flat plate.

It is important to note that (8) in the absence of a magnetic field $(M=0)$ and CNTs $(\phi=0)$ reduces to the same equation as obtained by Fang and Zhang [16] who provided an exact analytical solution of the famous Falkner-Skan equation for a conventional fluid when $\beta=-1$.

The skin friction coefficient $C_{f}$ and local Nusselt number $\mathrm{Nu}_{x}$ are defined as

$$
C=\frac{\tau_{w}}{\rho_{f} u_{e}^{2}}, \quad \mathrm{Nu}_{x}=\frac{x q_{w}}{k_{f}\left(T_{w}-T_{\infty}\right)},
$$

where $\tau_{w}$ and $q_{w}$ are the shear stress and heat flux at the wall which is given by

$$
\tau_{w}=\mu_{\mathrm{nf}}\left(\frac{\partial u}{\partial y}\right)_{y=0}, \quad q_{w}=-k_{\mathrm{nf}}\left(\frac{\partial T}{\partial y}\right)_{y=0} .
$$

Substituting (7) into (13) and (14), we obtain

$$
\begin{gathered}
\operatorname{Re}_{x}^{1 / 2} C_{f}=\sqrt{\frac{m+1}{2}} \frac{1}{(1-\phi)^{2.5}} f^{\prime \prime}(0), \\
\operatorname{Re}_{x}^{-1 / 2} \mathrm{Nu}_{x}=\sqrt{\frac{m+1}{2}} \frac{k_{\mathrm{nf}}}{k_{f}} \theta^{\prime}(0),
\end{gathered}
$$

where $\operatorname{Re}_{x}=u_{e} x / \nu_{f}$ is the local Reynolds number. 
TABLE 2: Comparison of skin friction $f^{\prime \prime}(0)$ when $M=\lambda=0$ for a conventional fluid $(\phi=0)$.

\begin{tabular}{lccccc}
\hline$m$ & White [38] & Yacob et al. [43] & Yih [44] & Khan and Pop [24] & Present results \\
\hline 0 & 0.4696 & 0.4696 & 0.4696 & 0.4696 & 0.4696 \\
$1 / 11$ & 0.6550 & 0.6550 & 0.6550 & 0.6550 & 0.6550 \\
$1 / 5$ & 0.8021 & 0.8021 & 0.8021 & 0.8021 & 0.8021 \\
$1 / 3$ & 0.9277 & 0.9277 & 0.9276 & 0.9277 & 0.9277 \\
$1 / 2$ & 1.0389 & 1.0389 & - & 1.0389 & 1.0389 \\
1 & 1.2326 & 1.2326 & 1.2326 & 1.2326 & 1.2326 \\
\hline
\end{tabular}

\section{Numerical Method}

Nonlinear ordinary differential equations (8) and (9) with boundary conditions (12) are solved by using an implicit finite difference method with a linearization technique. The details of this method are omitted here to save space but can be found in $[39,40]$. The step size is taken as $\Delta \eta=0.001$ and the convergence criteria are set to $10^{-6}$. The asymptotic boundary conditions given by (12) were replaced by using a value of 10 for the similarity variable $\eta_{\max }$ as follows:

$$
\eta_{\max }=10, \quad f^{\prime}(10)=1, \quad \theta(10)=0 .
$$

The choice of $\eta_{\max }=10$ ensures that all numerical solutions approached the asymptotic values correctly.

\section{Results and Discussion}

MHD flow of water-based carbon nanotubes (CNTs) over static and moving wedges is investigated numerically. The thermophysical properties of water and both CNTs are presented in Table 1 . The numerical results are compared in Tables 2 and 3 for skin friction and in Tables 4 and 5 for heat transfer in some special cases. The results are found to be in excellent agreement with the published data. The effects of the magnetic field and volume fraction of SWCNTs on the dimensionless velocity are displayed in Figures 2 and 3 for flow past horizontal and near the stagnation point of vertical stationary and moving flat plates, respectively. It can be seen that the hydrodynamic boundary layer thickness is larger in the absence of a magnetic field in each case. In fact, the magnetic field tends to reduce the boundary layer thickness. Figures 2(a) and 2(b) depict velocity profiles for the flow of SWCNTs over a stationary horizontal plate $(m=0)$ and near the stagnation point of a vertical stationary plate $(m=1)$, respectively. In the absence of magnetic field, the effect of volume fraction of SWCNTs on the dimensionless velocity can be noticed but as the magnetic field increases, its effect diminishes. When the horizontal and vertical plates move in the free stream direction, the dimensionless velocity profiles are shown in Figures 3(a) and 3(b), respectively. It is important to note that the boundary layer thickness is reduced in this case. The effects of volume fraction of CNTs are found to be negligible and the velocity boundary layer thickness reduces with an increase in magnetic field in both cases.
TABLE 3: Comparison of skin friction $f^{\prime \prime}(0)$ when $M=\lambda=0$ for a conventional fluid $(\phi=0)$.

\begin{tabular}{lccc}
\hline$\beta$ & Kuo [45] & Rajagopal et al. [46] & Present results \\
\hline 0 & 0.4696 & 0.4696 & 0.4696 \\
0.05 & 0.5317 & 0.5311 & 0.5311 \\
0.1 & 0.5879 & 0.5870 & 0.5870 \\
0.2 & 0.6876 & 0.6867 & 0.6867 \\
0.3 & 0.7755 & 0.7748 & 0.7748 \\
0.4 & 0.8549 & 0.8544 & 0.8544 \\
0.5 & 0.9279 & 0.9277 & 0.9277 \\
0.6 & 0.9958 & 0.9958 & 0.9958 \\
0.7 & 1.0594 & - & 1.0598 \\
0.8 & 1.1196 & 1.1203 & 1.1203 \\
0.9 & 1.1767 & - & 1.1777 \\
1 & 1.2313 & 1.2326 & 1.2326 \\
2 & 1.6831 & - & 1.6872 \\
\hline
\end{tabular}

TABLE 4: Comparison of dimensionless heat transfer $-\theta^{\prime}(0)$ when $M=\lambda=0$ for a conventional fluid $(\phi=0)$.

\begin{tabular}{ccccc}
\hline$m$ & Kuo [45] & Blasius [47] & Khan and Pop [24] & Present results \\
\hline 0 & 0.8673 & 0.8673 & 0.8769 & 0.8769 \\
1 & 1.1147 & 1.1152 & 1.1279 & 1.1280 \\
\hline
\end{tabular}

The effects of magnetic field and volume fraction of SWCNTs are illustrated in Figure 4 for stationary plates and in Figure 5 for moving plates in opposite direction to free stream, respectively. In each case, the magnetic field tends to reduce the thermal boundary layer thickness. The comparison of Figures 4(a) and 4(b) shows that the thermal boundary layer thickness is smaller in the case of flow over a horizontal plate and hence less thermal resistance is offered in this case. However, when the plates are moving in the direction of the free stream, the magnetic field has no appreciable effect on the thermal boundary layer thickness (as shown in Figures 5(a) and 5(b)). Conventional fluids (phi = 0 ) have a smaller thermal boundary layer thickness and as the volume fraction of SWCNTs increases, the thermal boundary 
TABLE 5: Comparison of dimensionless heat transfer $-\theta^{\prime}(0)$ when $M=\lambda=0$ for a conventional fluid $(\phi=0)$.

\begin{tabular}{lccccccccc}
\hline Pr & \multicolumn{3}{c}{$\beta=0$} & & \multicolumn{3}{c}{$\beta=0.3$} & & \multicolumn{2}{c}{$\beta=1$} \\
& White [38] & Kuo [45] & Present results & White [38] & Kuo [45] & Present results & White [38] & Kuo [45] & Present results \\
\hline 1 & 0.4696 & 0.4696 & 0.4696 & 0.5195 & 0.5200 & 0.5195 & 0.5705 & 0.5708 & 0.5705 \\
2 & 0.5972 & 0.5972 & 0.5972 & 0.6691 & 0.6696 & 0.6690 & 0.7437 & 0.7441 & 0.7437 \\
3 & 0.6860 & 0.6860 & 0.6860 & 0.7734 & 0.7741 & 0.7734 & 0.8652 & 0.8657 & 0.8652 \\
6 & 0.8673 & 0.8673 & 0.8673 & 0.9873 & 0.9881 & 0.9873 & 1.1147 & 1.1152 & 1.1147 \\
10 & 1.0297 & 1.0297 & 1.0297 & 1.1791 & 1.1800 & 1.1791 & 1.3388 & 1.3394 & 1.3388 \\
30 & 1.4873 & 1.4873 & 1.4873 & 1.7198 & 1.7210 & 1.7198 & 1.9706 & 1.9714 & 1.9706 \\
60 & 1.8746 & 1.8746 & 1.8746 & 2.1776 & 2.1791 & 2.1776 & 2.5054 & 2.5063 & 2.5054 \\
100 & 2.2229 & 2.2229 & 2.2229 & 2.5892 & 2.5910 & 2.5892 & 2.9863 & 2.9874 & 2.9863 \\
\hline
\end{tabular}

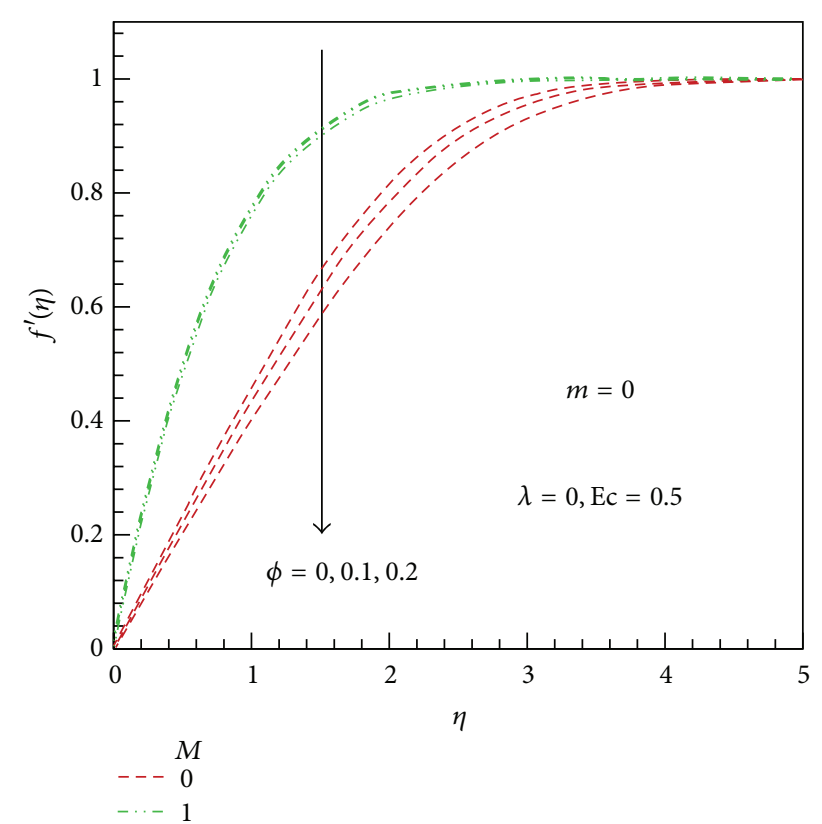

(a)

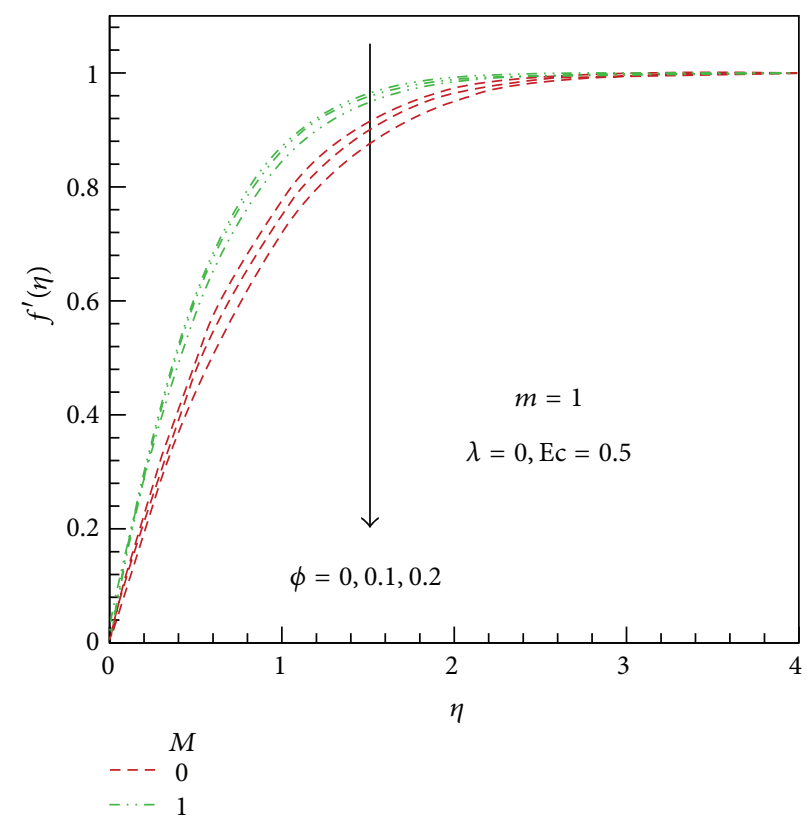

(b)

FIGURE 2: Effect of magnetic field on dimensionless velocity of water-based SWCNTs for (a) flow past horizontal and (b) flow near the stagnation point of vertical stationary flat plates.

layer thickness also increases due to an increase in the density of CNTs.

The variation of skin friction with magnetic field and volume fraction of CNTs is presented in Figure 6 for the horizontal plate moving in an opposite direction to the free stream. In Figure 6(a), SWCNTs are used whereas in Figure 6(b) MWCNTs are used to show the comparison of skin friction in the presence of a magnetic field. It is noticed that the skin friction increases with volume fraction of CNTs in both cases. However, skin friction of SWCNTs is found to be higher than MWCNTs. It is due to higher density of SWCNTs than MWCNTs (Table 1). The effect of magnetic field creates a Lorentz force which acts against the flow and thus enhances the skin friction. As the horizontal plate moves in the opposing flow, the hydraulic resistance also increases which helps in an increase in skin friction. When the horizontal plate moves in an assisting flow, Figures 7(a) and 7(b) show the variation of skin friction with volume fraction of CNTs and magnetic field. In this case, the resistance decreases and hence the skin friction decreases with an increase in the plate velocity. The same behavior was observed when a vertical plate moves in opposing flow. For a vertical plate moving in opposite direction to the free stream, the variation of skin friction with magnetic field and volume fraction of CNTs is illustrated in Figure 8 for both CNTs in the presence of a magnetic field. Similar to the horizontal plate, skin friction also increases with plate velocity as shown in Figures 8(a) and $8(\mathrm{~b})$. Figures $9(\mathrm{a})$ and 9 (b) present the variation of skin friction of CNTs along wedges moving in opposite direction to the free stream in the presence of a magnetic field. Due to 


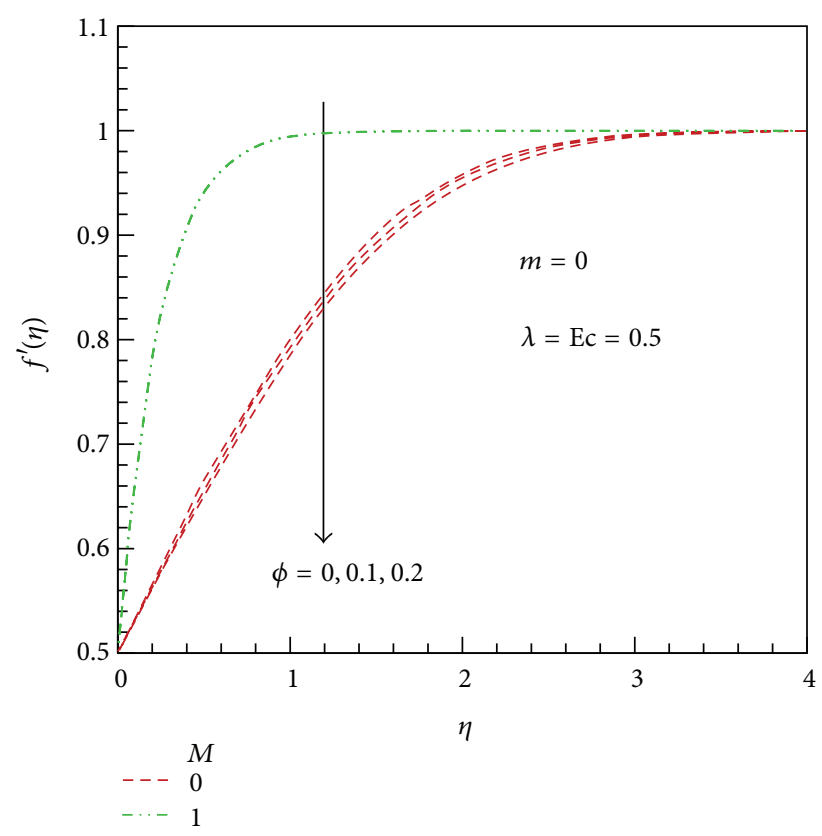

(a)

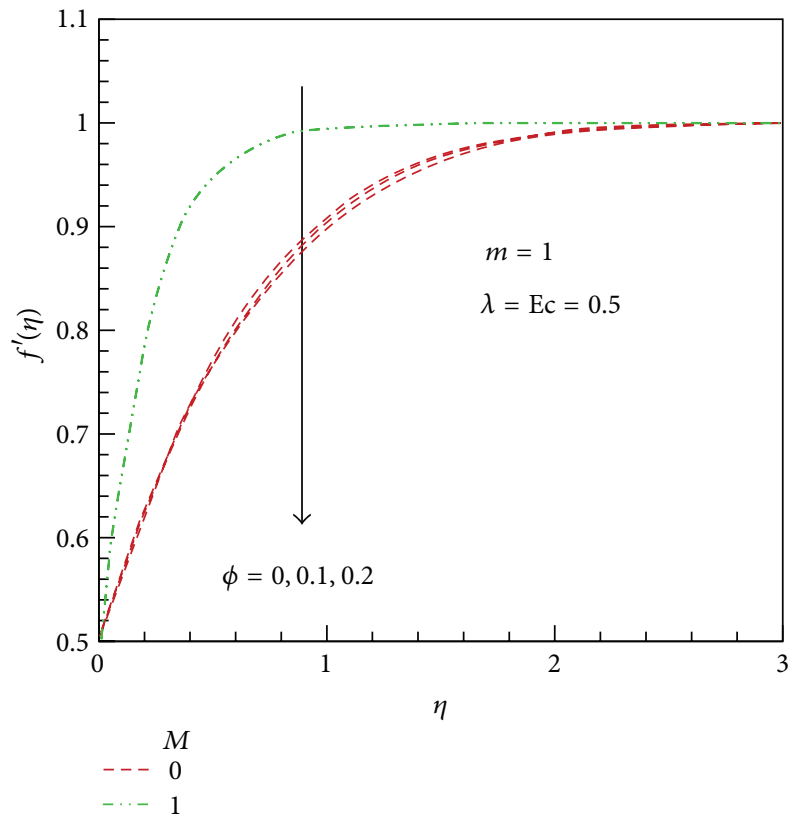

(b)

FIGURE 3: Effect of magnetic field on dimensionless velocity of water-based nanofluids for (a) flow past horizontal and (b) flow near the stagnation point of vertical flat plates moving in the same direction.

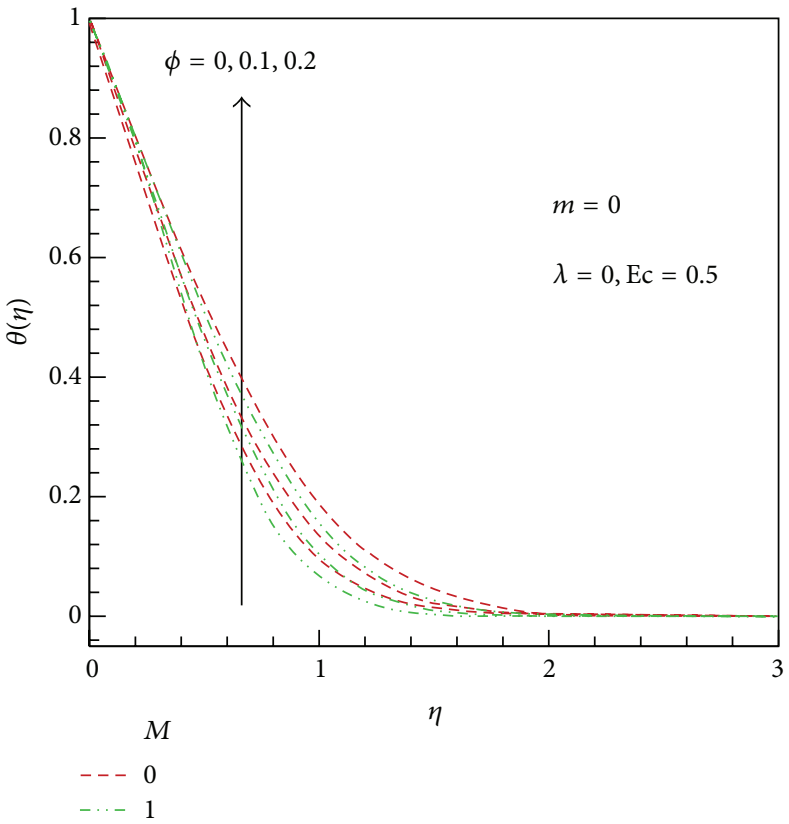

(a)

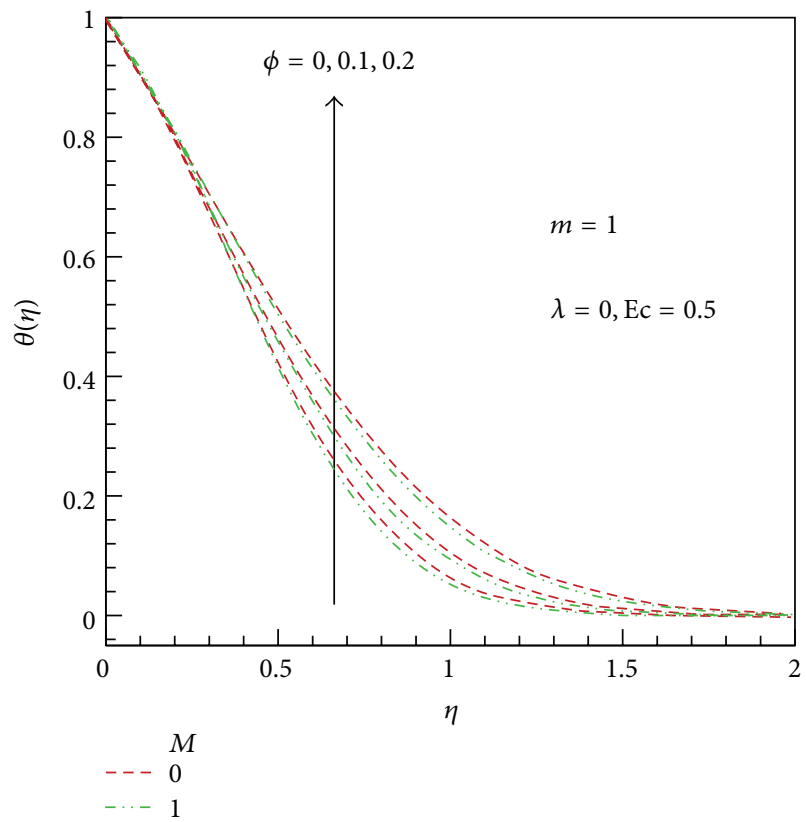

(b)

FIGURE 4: Effect of magnetic field on dimensionless temperature of water-based nanofluids for (a) flow past horizontal and (b) flow near the stagnation point of vertical stationary flat plates.

the increase in density with volume fraction of CNTs, the skin friction increases with volume friction of CNTs and wedge parameter. For conventional fluid (water) the skin friction is the same for both CNTs. Again, the skin friction is found to be higher for SWCNTs for both wedges.
The variation of Nusselt numbers with volume fraction of CNTs, magnetic and wedge parameters is reported in Figures 10-13 for special cases. Due to higher thermal conductivity, SWCNTs possess higher Nusselt numbers in each case. The effects of magnetic field and the motion 


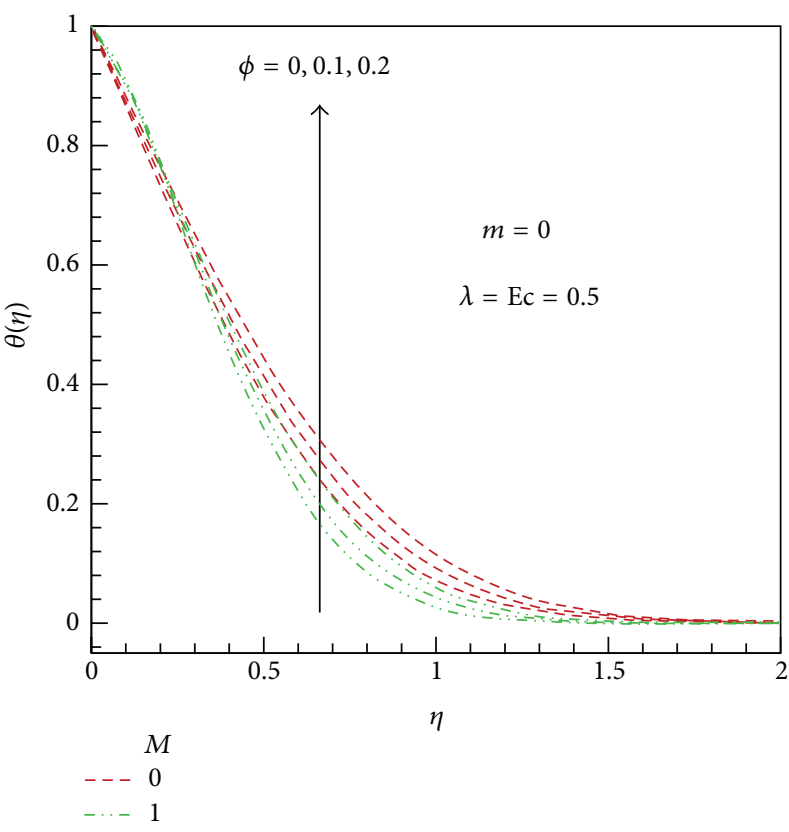

(a)

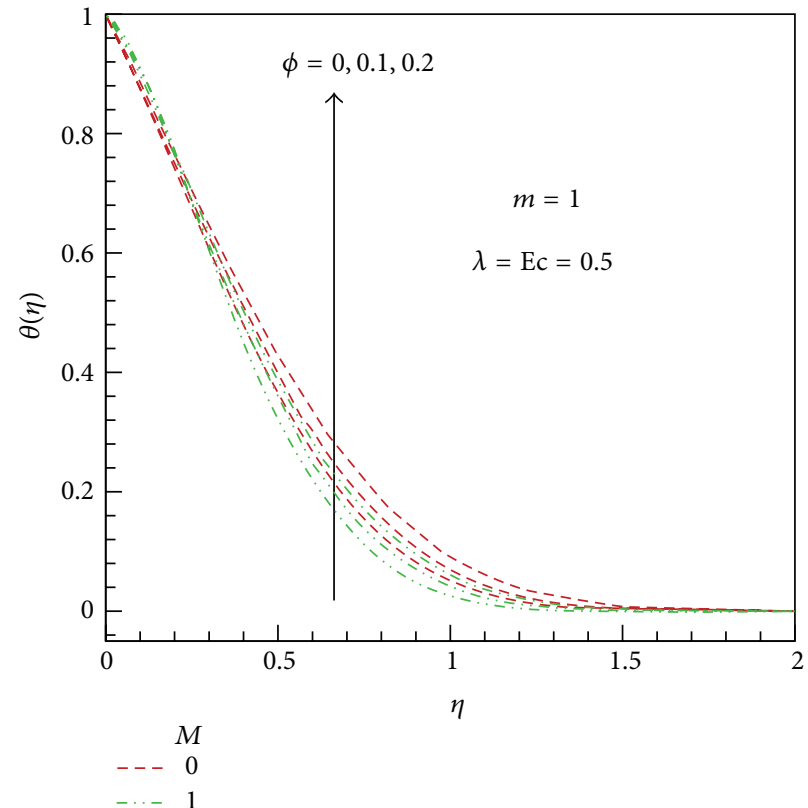

(b)

FIGURE 5: Effect of magnetic field on dimensionless temperature of water-based nanofluids for (a) flow past horizontal and (b) flow near the stagnation point of vertical flat plates moving in the same direction.

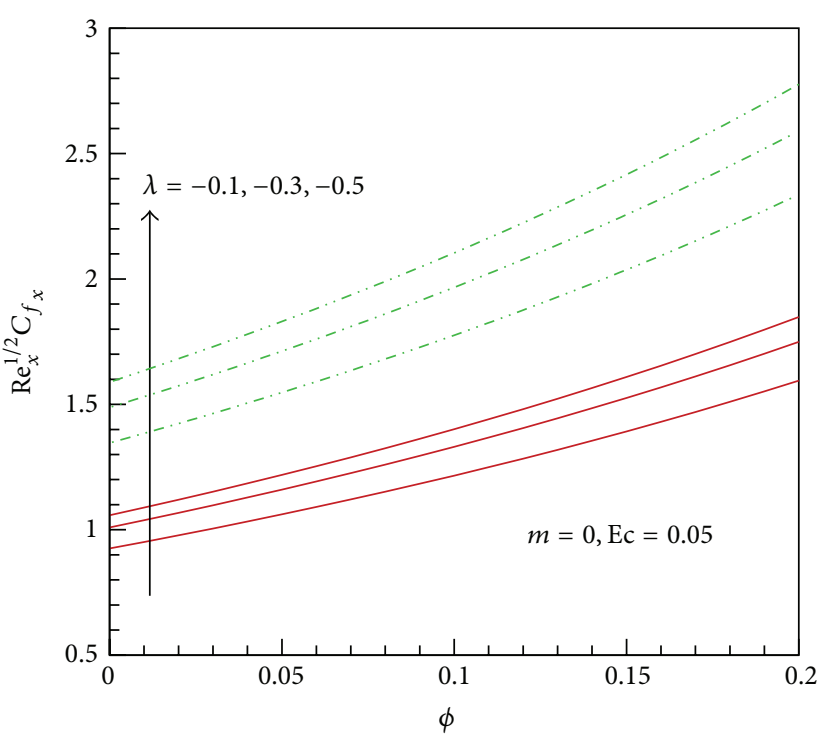

$$
\begin{gathered}
M \\
-1 \\
\hline
\end{gathered}
$$

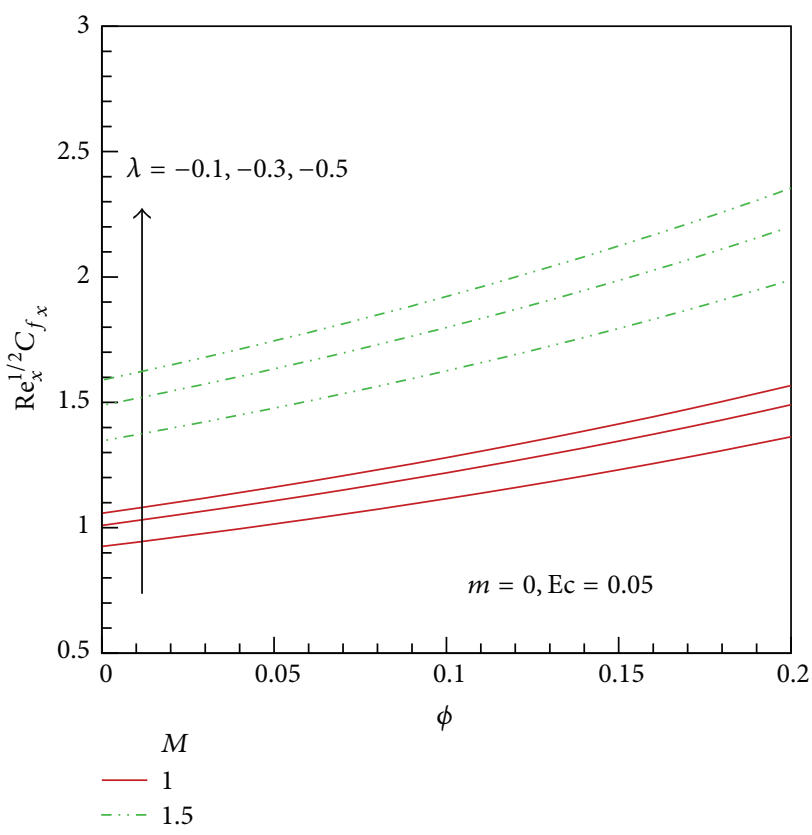

(b) MWCNT

(a) SWCNT

FIGURE 6: Variation of skin friction with volume fraction of nanoparticles and magnetic field along a horizontal plate moving in opposite direction to the free stream for (a) SWCNTs and (b) MWCNTs.

of the horizontal plate in the direction opposite to the free stream are shown in Figure 10(a) for SWCNTs and in Figure 10(b) for MWCNTs. It is noticed that Nusselt numbers decrease with an increase in the plate velocity. However, magnetic field helps in increasing Nusselt numbers for both CNTs. The same behavior can be observed in Figure 11 for a vertical plate and in Figure 12 for a wedge moving in opposite direction to the free stream. However, when 

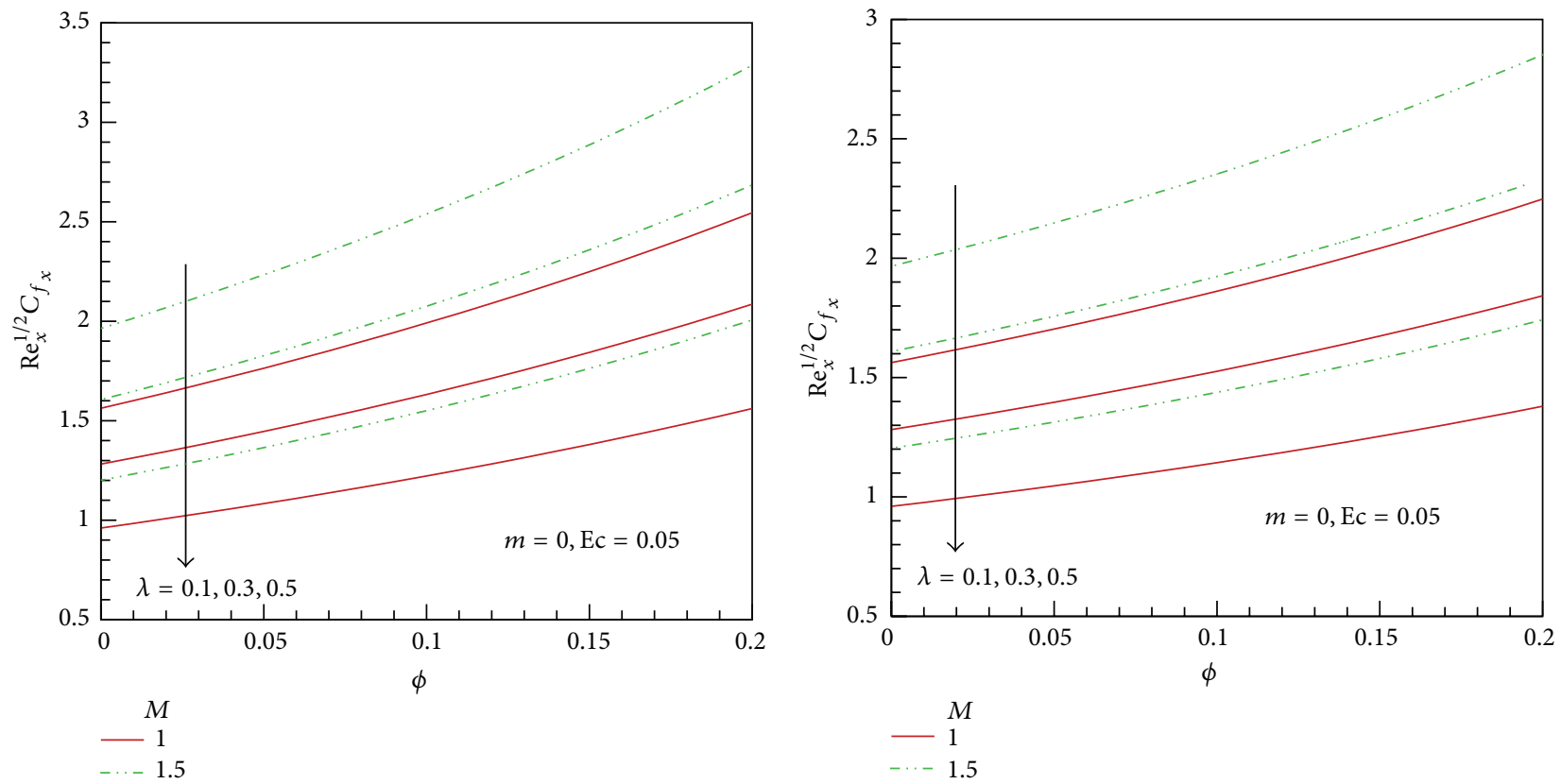

(a) SWCNT

(b) MWCNT

FiguRE 7: Variation of skin friction with volume fraction of nanoparticles and magnetic field along a horizontal plate moving in the same direction to the free stream for (a) SWCNTs and (b) MWCNTs.

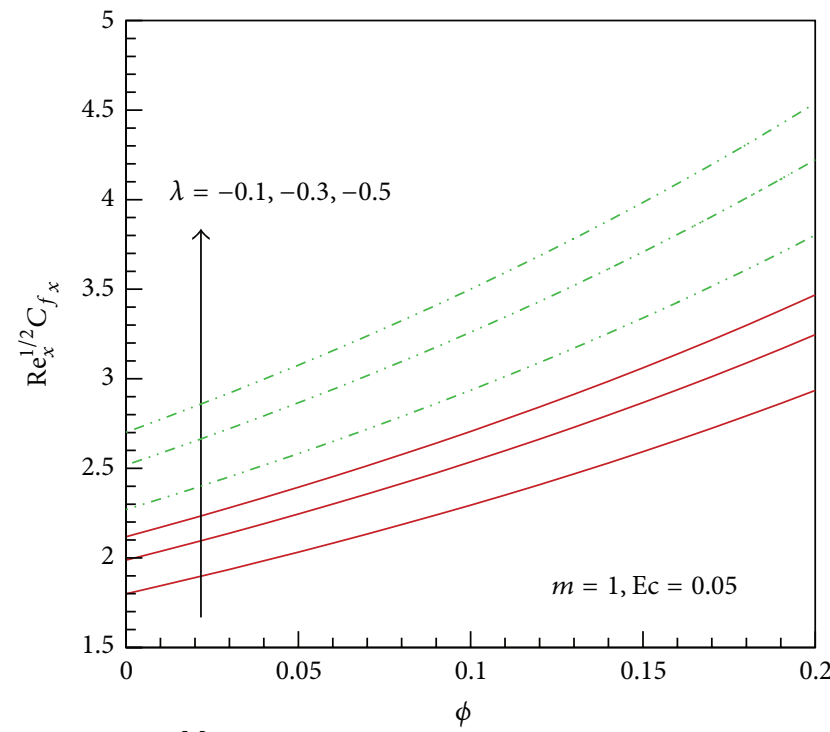

$$
\begin{array}{rl} 
& M \\
-\cdots & 1 \\
-\cdot-5 & 1.5
\end{array}
$$

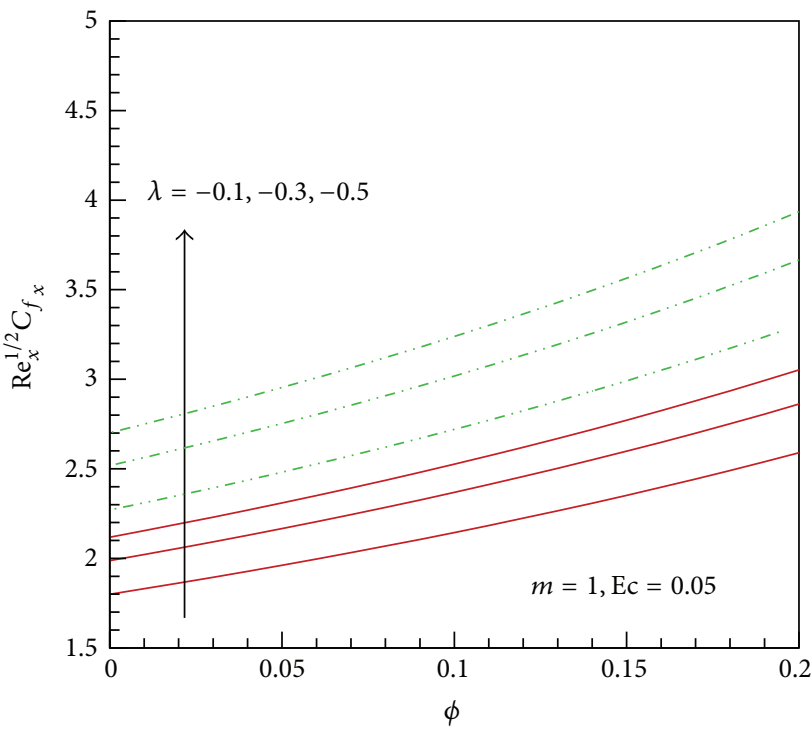

$$
\begin{gathered}
M \\
-1 \\
-\cdots-1.5
\end{gathered}
$$

(b) MWCNT

FIGURE 8: Variation of skin friction with volume fraction of nanoparticles and magnetic field along a vertical plate moving in opposite direction to the free stream for (a) SWCNTs and (b) MWCNTs.

the horizontal plate moves in the direction of free stream, the Nusselt numbers increase with plate velocity. This is shown in Figure 13(a) for SWCNTs and in Figure 13(b) for MWCNTs.

\section{Conclusions}

The steady MHD flow and heat transfer from water-based CNTs over a static/moving wedge are studied numerically. 


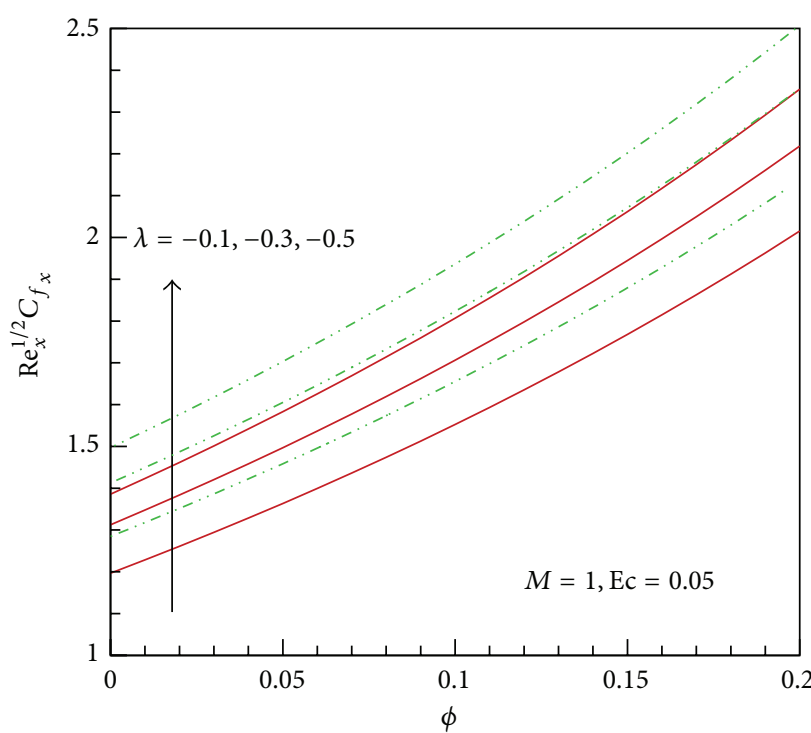

$\begin{array}{ll}m & m \\ -\cdots- & 1 / 6\end{array}$

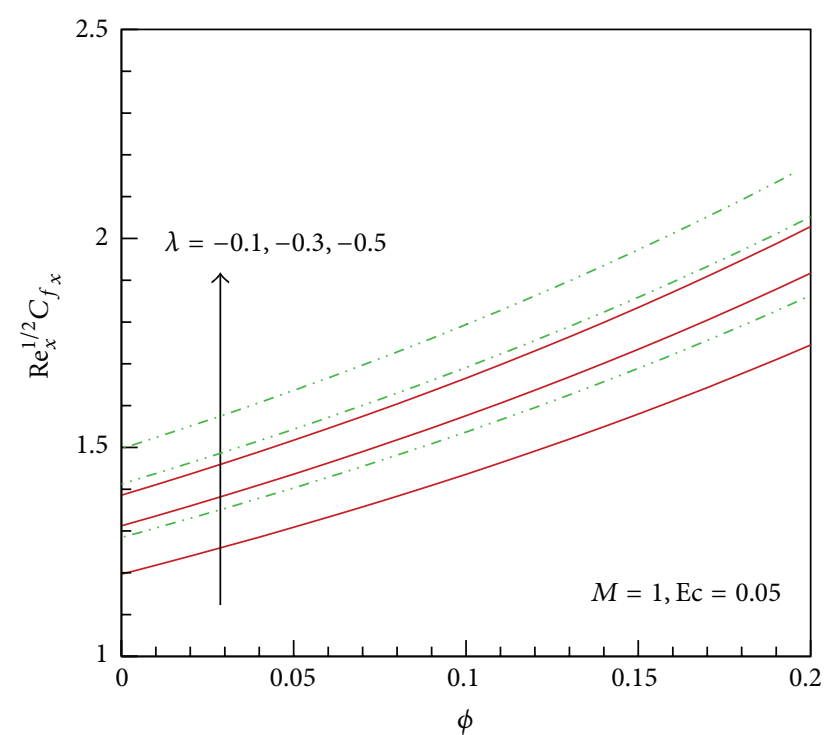

$m$
$-1 / 6$

(a) SWCNT

(b) MWCNT

FIGURE 9: Variation of skin friction with volume fraction of nanoparticles and wedge parameter along a wedge moving in opposite direction to the free stream for (a) SWCNTs and (b) MWCNTs.

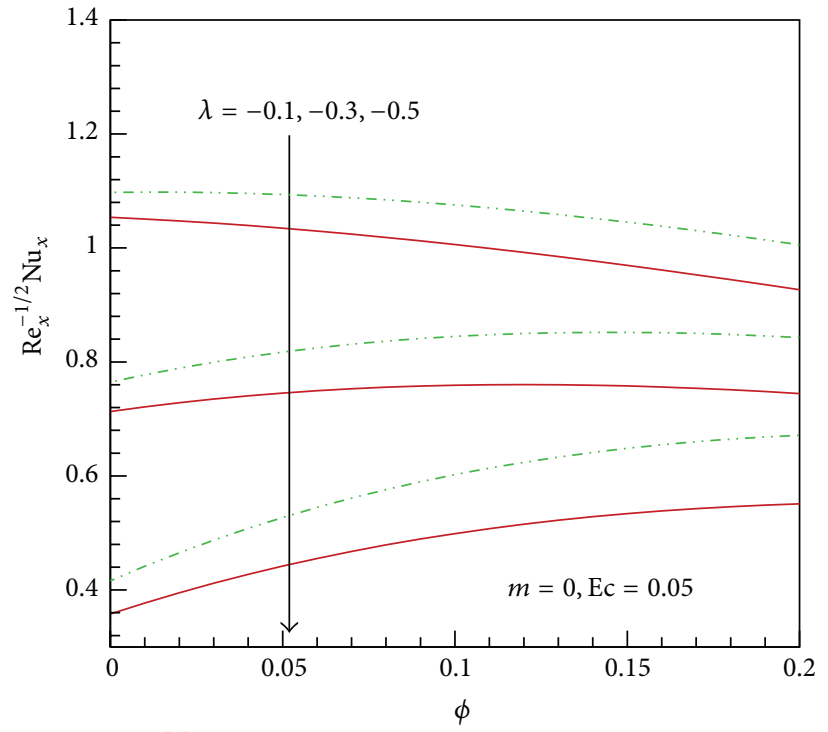
$M$
-1
$-\cdots-1.5$

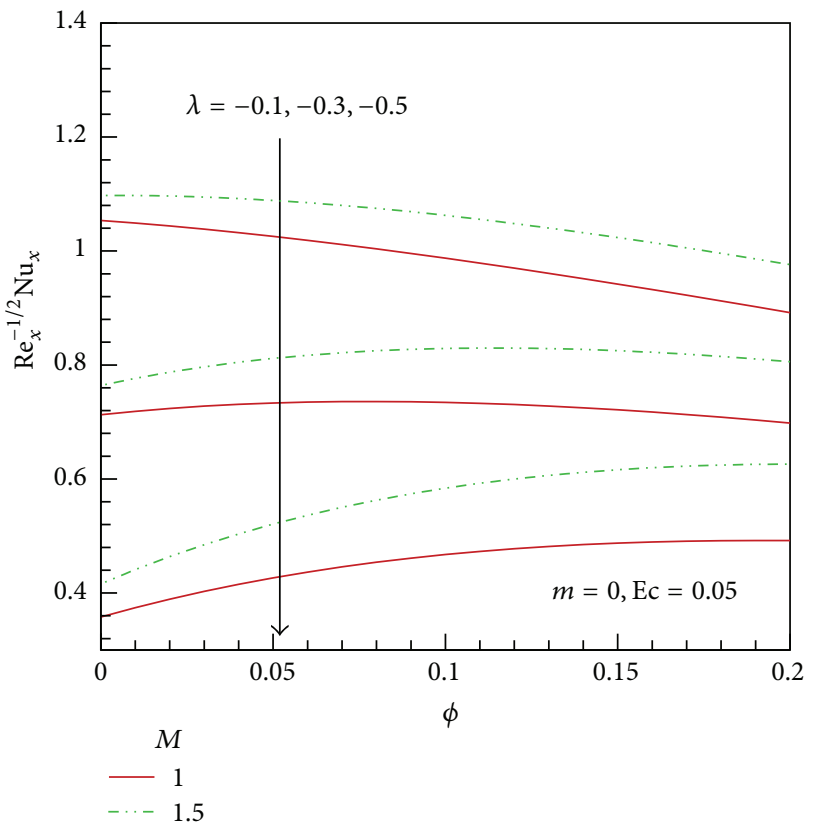

(b) MWCNT

(a) SWCNT

FIGURE 10: Variation of Nusselt number with volume fraction of nanoparticles and magnetic field along a horizontal plate moving in opposite direction to the free stream for (a) SWCNTs and (b) MWCNTs.

This problem reduces to the classical Falkner-Skan's [1] problem of the boundary layer flow of a viscous (Newtonian) fluid past a fixed wedge, when $M=\phi=0$. The effects of volume fraction of CNTs, magnetic and wedge parameters are investigated and presented graphically. It is found that the following happens.

(i) A magnetic field increases skin friction and heat transfer rates. 


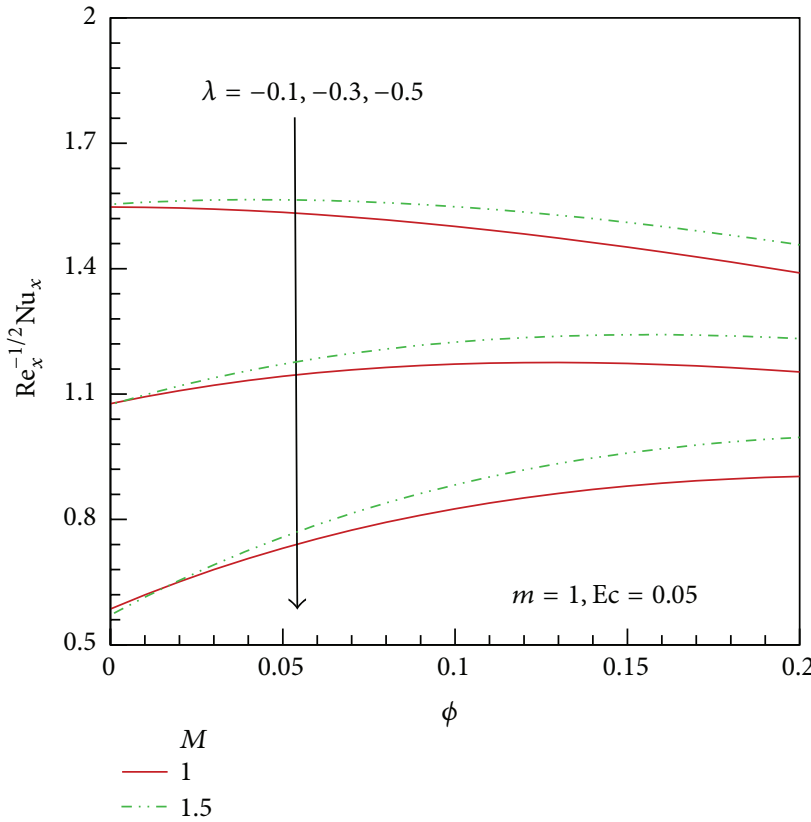

(a) SWCNT

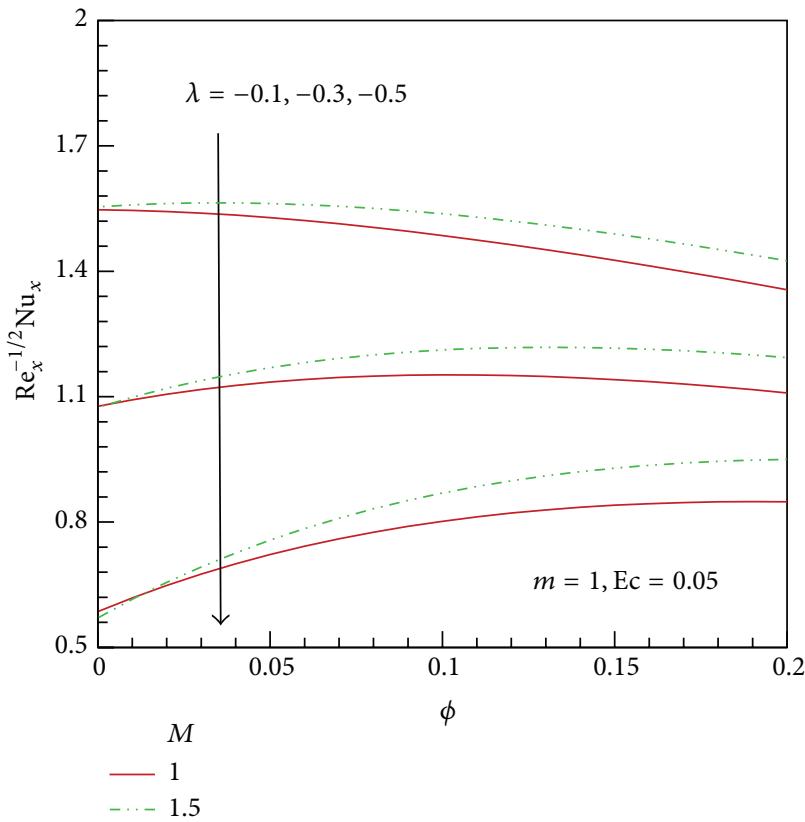

(b) MWCNT

FIGURE 11: Variation of Nusselt number with volume fraction of nanoparticles and magnetic field along a vertical plate moving in opposite direction to the free stream for (a) SWCNTs and (b) MWCNTs.

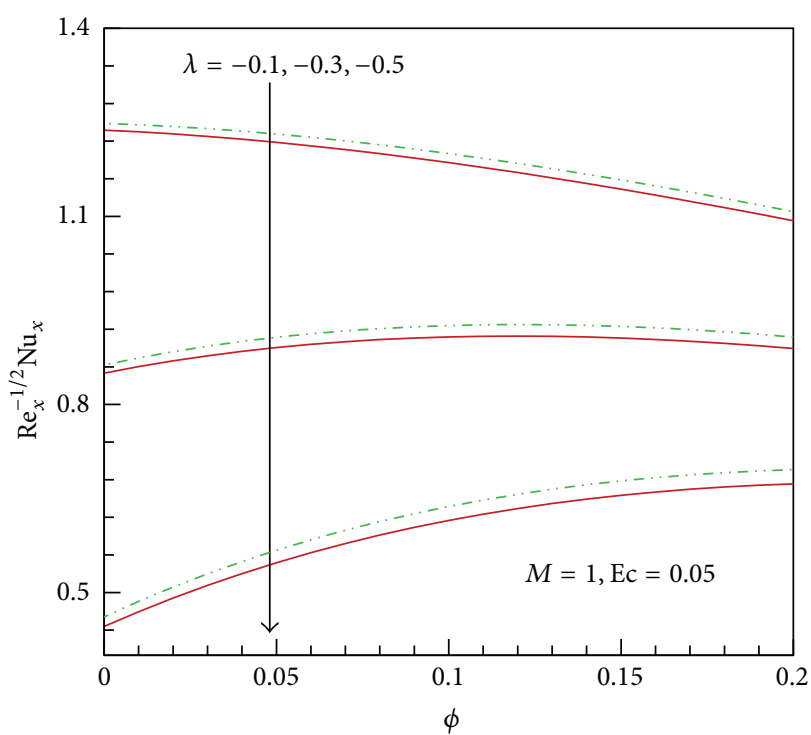
$\begin{array}{ll}m \\ -\cdots- & 1 / 6\end{array}$

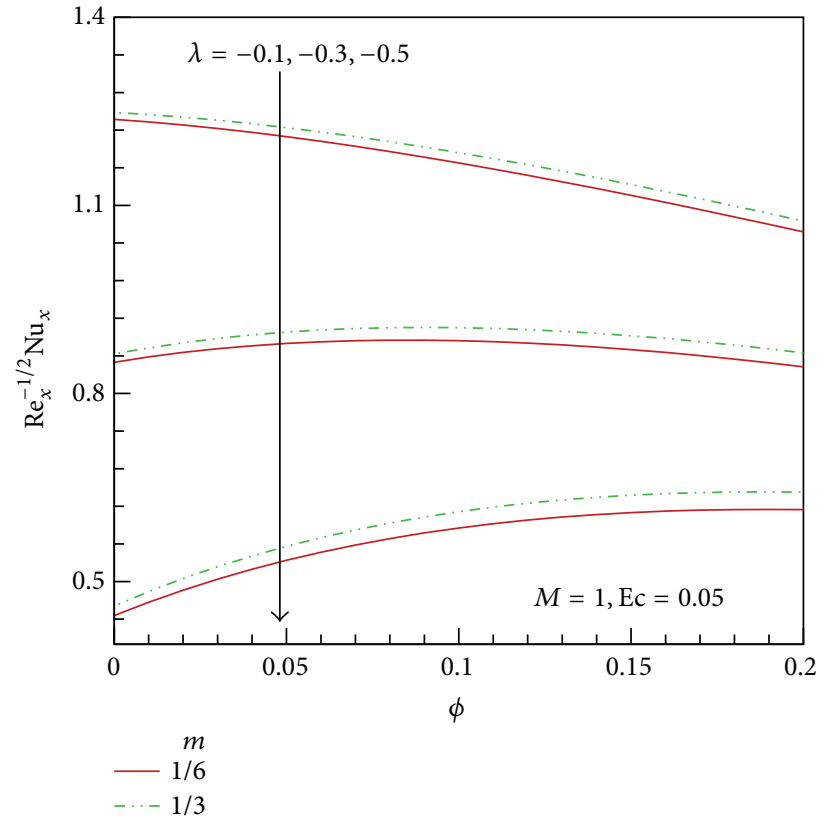

(b) MWCNT

FIGURE 12: Variation of Nusselt number with volume fraction of nanoparticles and wedge parameter along a wedge moving in opposite direction to the free stream for (a) SWCNTs and (b) MWCNTs.

(ii) Velocity boundary layer thickness is smaller for the flow near the stagnation point of vertical stationary/moving flat plates.

(iii) Thermal boundary layer thickness is smaller for the flow past horizontal stationary/moving flat plates. (iv) MWCNTs offer less friction in each case.

(v) Heat transfer rates increase when plates move in the free stream direction.

(vi) Heat transfer rates decrease when plates move in opposite direction to the free stream. 


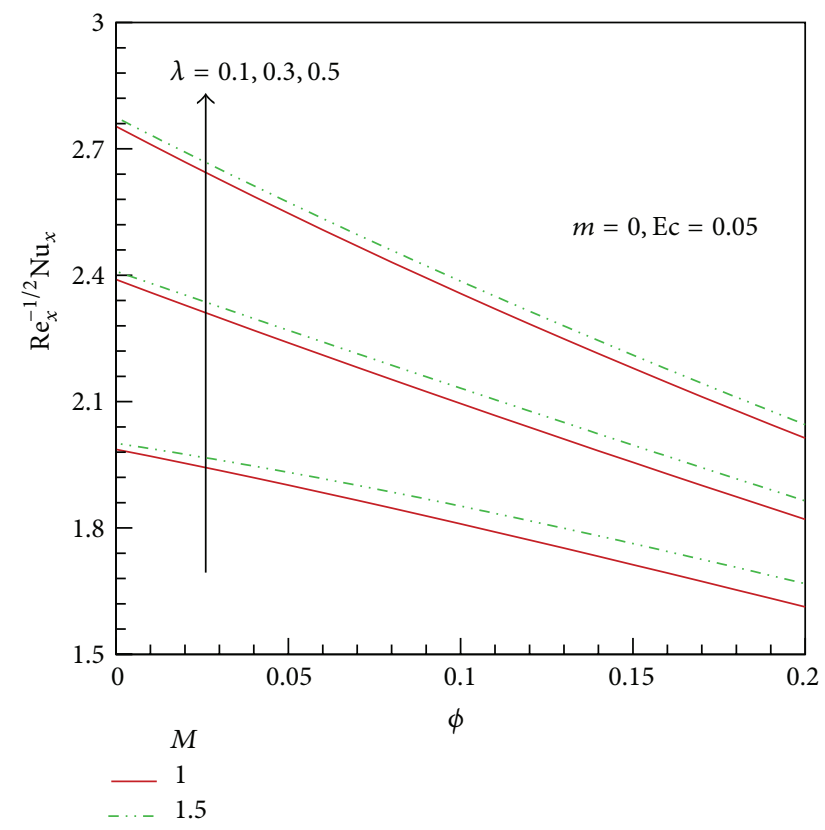

(a) SWCNT

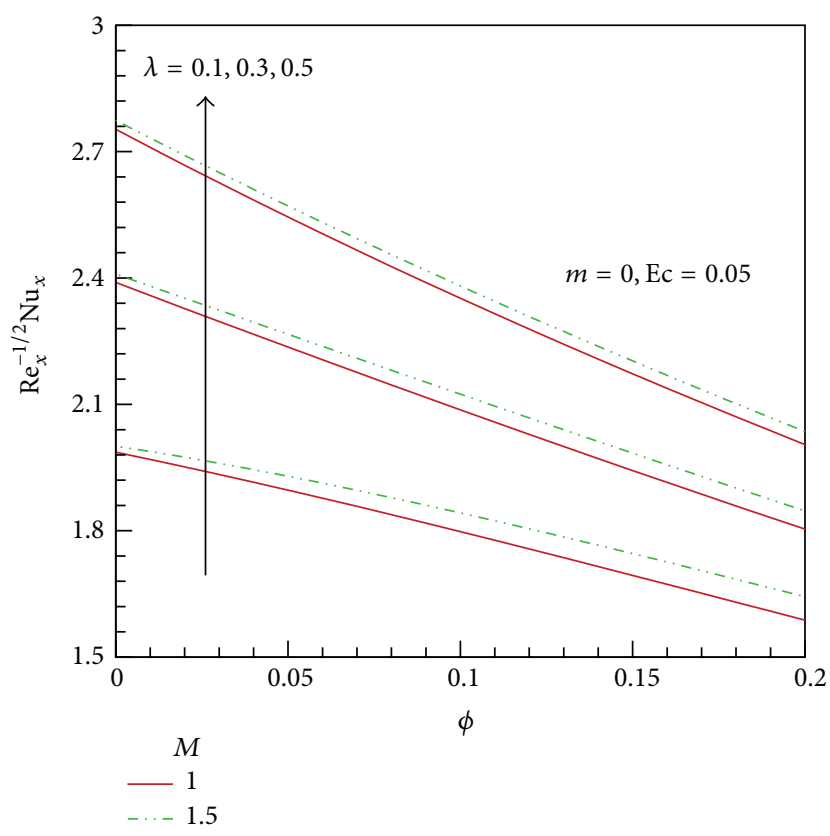

(b) MWCNT

FIGURE 13: Variation of Nusselt number with volume fraction of nanoparticles and magnetic field along a horizontal plate moving in the same direction to the free stream for (a) SWCNT and (b) MWCNT.

\section{Conflict of Interests}

The authors declare that there is no conflict of interests regarding the publication of this paper.

\section{References}

[1] V. M. Falkner and S. W. Skan, "Some approximate solutions of the boundary layer equations," Philosophical Magazine, vol. 12, no. 80 , pp. 865-896, 1931.

[2] D. R. Hartree, "On an equation occurring in Falkner and Skan's approximate treatment of the equations of the boundary layer," Mathematical Proceedings of the Cambridge Philosophical Society, vol. 33, no. 2, pp. 223-239, 1937.

[3] J. C. Y. Koh and J. P. Hartnett, "Skin friction and heat transfer for incompressible laminar flow over porous wedges with suction and variable wall temperature," International Journal of Heat and Mass Transfer, vol. 2, no. 3, pp. 185-198, 1961.

[4] H. S. Takhar and I. Pop, "On MHD heat transfer from a wedge at large prandtl numbers," Mechanics Research Communications, vol. 11, no. 3, pp. 191-194, 1984.

[5] H.-T. Lin and L.-K. Lin, "Similarity solutions for laminar forced convection heat transfer from wedges to fluids of any Prandtl number," International Journal of Heat and Mass Transfer, vol. 30, no. 6, pp. 1111-1118, 1987.

[6] T. Watanabe, "Thermal boundary layers over a wedge with uniform suction or injection in forced flow," Acta Mechanica, vol. 83, no. 3-4, pp. 119-126, 1990.

[7] K. A. Yih, "MHD forced convection flow adjacent to a nonisothermal wedge," International Communications in Heat and Mass Transfer, vol. 26, no. 6, pp. 819-827, 1999.

[8] A. Hossain, S. Munir, and D. A. S. Rees, "Flow of viscous incompressible fluid with temperature dependent viscosity and thermal conductivity past a permeable wedge with uniform surface heat flux," International Journal of Thermal Sciences, vol. 39, no. 6, pp. 635-644, 2000.

[9] D. Pal and H. Mondal, "Influence of temperature-dependent viscosity and thermal radiation on MHD forced convection over a non-isothermal wedge," Applied Mathematics and Computation, vol. 212, no. 1, pp. 194-208, 2009.

[10] A. Ishak, R. Nazar, and I. Pop, "MHD boundary-layer flow of a micropolar fluid past a wedge with constant wall heat flux," Communications in Nonlinear Science and Numerical Simulation, vol. 14, no. 1, pp. 109-118, 2009.

[11] X. Su, L. Zheng, X. Zhang, and J. Zhang, "MHD mixed convective heat transfer over a permeable stretching wedge with thermal radiation and ohmic heating," Chemical Engineering Science, vol. 78, pp. 1-8, 2012.

[12] K. V. Prasad, P. S. Datti, and K. Vajravelu, "MHD mixed convection flow over a permeable non-isothermal wedge," Journal of King Saud University-Science, vol. 25, no. 4, pp. 313324, 2013.

[13] A. T. M. M. Rahman, M. S. Alam, M. A. Alim, and M. K. Chowdhury, "Unsteady MHD forced convective heat and mass transfer flow along a wedge with variable electric conductivity and thermophoresis," Procedia Engineering, vol. 5, pp. 531-537, 2013.

[14] I. Muhaimin, R. Kandasamy, A. B. Khamis, and R. Roslan, "Effect of thermophoresis particle deposition and chemical reaction on unsteady MHD mixed convective flow over a porous wedge in the presence of temperature-dependent viscosity," Nuclear Engineering and Design, vol. 261, pp. 95-106, 2013.

[15] S. Abbasbandy and T. Hayat, "Solution of the MHD FalknerSkan flow by homotopy analysis method," Communications in Nonlinear Science and Numerical Simulation, vol. 14, no. 9-10, pp. 3591-3598, 2009. 
[16] T. Fang and J. Zhang, "An exact analytical solution of the Falkner-Skan equation with mass transfer and wall stretching," International Journal of Non-Linear Mechanics, vol. 43, no. 9, pp. 1000-1006, 2008.

[17] U. S. Choi, "Enhancing thermal conductivity of fluids with nanoparticles," in Proceedings of the ASME Fluids Engineering Division, vol. 231, pp. 99-103, San Francisco, Calif, USA, November 1995.

[18] D. A. Nield and A. V. Kuznetsov, "The Cheng-Minkowycz problem for natural convective boundary-layer flow in a porous medium saturated by a nanofluid," International Journal of Heat and Mass Transfer, vol. 52, no. 25-26, pp. 5792-5795, 2009.

[19] A. V. Kuznetsov and D. A. Nield, "Double-diffusive natural convective boundary-layer flow of a nanofluid past a vertical plate," International Journal of Thermal Sciences, vol. 50, no. 5, pp. 712-717, 2011.

[20] D. A. Nield and A. V. Kuznetsov, "The Cheng-Minkowycz problem for the double-diffusive natural convective boundary layer flow in a porous medium saturated by a nanofluid," International Journal of Heat and Mass Transfer, vol. 54, no. 1-3, pp. 374-378, 2011.

[21] P. Cheng and W. J. Minkowycz, "Free convection about a vertical flat plate embedded in a porous medium with application to heat transfer from a dike," Journal of Geophysical Research, vol. 82, no. 14, pp. 2040-2044, 1977.

[22] A. V. Kuznetsov and D. A. Nield, "Natural convective boundarylayer flow of a nanofluid past a vertical plate," International Journal of Thermal Sciences, vol. 49, no. 2, pp. 243-247, 2010.

[23] M. Narayana and P. Sibanda, "Laminar flow of a nanoliquid film over an unsteady stretching sheet," International Journal of Heat and Mass Transfer, vol. 55, no. 25-26, pp. 7552-7560, 2012.

[24] W. A. Khan and I. Pop, "Boundary layer flow past a wedge moving in a nanofluid," Mathematical Problems in Engineering, vol. 2013, Article ID 637285, 7 pages, 2013.

[25] N. A. Yacob, A. Ishak, R. Nazar, and I. Pop, "Falkner-Skan problem for a static and moving wedge with prescribed surface heat flux in a nanofluid," International Communications in Heat and Mass Transfer, vol. 38, no. 2, pp. 149-153, 2011.

[26] M. M. Rahman, M. A. Al-Lawatia, I. A. Eltayeb, and N. Al-Salti, "Hydromagnetic slip flow of water based nanofluids past a wedge with convective surface in the presence of heat generation (or) absorption," International Journal of Thermal Sciences, vol. 57, pp. 172-182, 2012.

[27] M. M. Rahman, W. A. Al-Mazroui, F. S. Al-Hatmi, M. A. AlLawatia, and I. A. Eltayeb, "The role of a convective surface in models of the radiative heat transfer in nanofluids," Nuclear Engineering and Design, vol. 275, pp. 382-392, 2014.

[28] A. J. Chamkha, S. Abbasbandy, A. M. Rashad, and K. Vajravelu, "Radiation effects on mixed convection over a wedge embedded in a porous medium filled with a nanofluid," Transport in Porous Media, vol. 91, no. 1, pp. 261-279, 2012.

[29] P. K. Kameswaran, M. Narayana, S. Shaw, and P. Sibanda, "Heat and mass transfer from an isothermal wedge in nanofluids with Soret effect," The European Physical Journal Plus, vol. 129, no. 7, pp. 154-164, 2014.

[30] S. Halelfadl, T. Maré, and P. Estellé, "Efficiency of carbon nanotubes water based nanofluids as coolants," Experimental Thermal and Fluid Science, vol. 53, pp. 104-110, 2014.

[31] T. Maré, S. Halelfadl, O. Sow, P. Estellé, S. Duret, and F. Bazantay, "Comparison of the thermal performances of two nanofluids at low temperature in a plate heat exchanger," Experimental Thermal and Fluid Science, vol. 35, no. 8, pp. 1535-1543, 2011.
[32] M.-S. Liu, M. Ching-Cheng Lin, I.-T. Huang, and C.-C. Wang, "Enhancement of thermal conductivity with carbon nanotube for nanofluids," International Communications in Heat and Mass Transfer, vol. 32, no. 9, pp. 1202-1210, 2005.

[33] H. Xie, H. Lee, W. Youn, and M. Choi, "Nanofluids containing multiwalled carbon nanotubes and their enhanced thermal conductivities," Journal of Applied Physics, vol. 94, no. 8, pp. 4967-4971, 2003.

[34] Y. Ding, H. Alias, D. Wen, and R. A. Williams, "Heat transfer of aqueous suspensions of carbon nanotubes (CNT nanofluids)," International Journal of Heat and Mass Transfer, vol. 49, no. 1-2, pp. 240-250, 2006.

[35] S. Halelfadl, P. Estellé, and T. Maré, "Heat transfer properties of aqueous carbon nanotubes nanofluids in coaxial heat exchanger under laminar regime," Experimental Thermal and Fluid Science, vol. 55, pp. 174-180, 2014.

[36] W. A. Khan, Z. H. Khan, and M. Rahi, "Fluid flow and heat transfer of carbon nanotubes along a flat plate with Navier slip boundary," Applied Nanoscience, vol. 4, no. 5, pp. 633-641, 2014.

[37] R. Ul Haq, Z. H. Khan, and W. A. Khan, "Thermophysical effects of carbon nanotubes on MHD flow over a stretching surface," Physica E: Low-dimensional Systems and Nanostructures, vol. 63, pp. 215-222, 2014.

[38] F. M. White, Viscous Fluid Flow, McGraw-Hill, NewYork, NY, USA, 2nd edition, 1991.

[39] R. E. Bellman and R. E. Kalaba, Quasilinearization and NonLinear Boundary Value Problems, American Elsevier Publishing, New York, NY, USA, 1965.

[40] K. Inouye and A. Tate, "Finite differences version of quasilinearization applied to boundary layer equations," AIAA Journal, vol. 12, no. 4, pp. 558-560, 1974.

[41] J. Hone, "Carbon nanotubes: thermal properties," in Dekker Encyclopedia of Nanoscience and Nanotechnology, pp. 603-610, 2004.

[42] R. S. Ruoff and D. C. Lorents, "Mechanical and thermal properties of carbon nanotubes," Carbon, vol. 33, no. 7, pp. 925930, 1995.

[43] N. A. Yacob, A. Ishak, and I. Pop, "Falkner-Skan problem for a static or moving wedge in nanofluids," International Journal of Thermal Sciences, vol. 50, no. 2, pp. 133-139, 2011.

[44] K. A. Yih, "Uniform suction/blowing effect on forced convection about a wedge: uniform heat flux," Acta Mechanica, vol. 128, no. 3-4, pp. 173-181, 1998.

[45] B.-L. Kuo, "Heat transfer analysis for the Falkner-Skan wedge flow by the differential transformation method," International Journal of Heat and Mass Transfer, vol. 48, no. 23-24, pp. 50365046, 2005.

[46] K. R. Rajagopal, A. S. Gupta, and T. Y. Na, "A note on the Falkner-Skan flows of a non-Newtonian fluid," International Journal of Non-Linear Mechanics, vol. 18, no. 4, pp. 313-320, 1983.

[47] H. Blasius, "Grenzschichten in Flussigkeiten mit kleiner Reibung," Zentralblatt Mathematical Physics, vol. 56, pp. 1-37, 1908. 

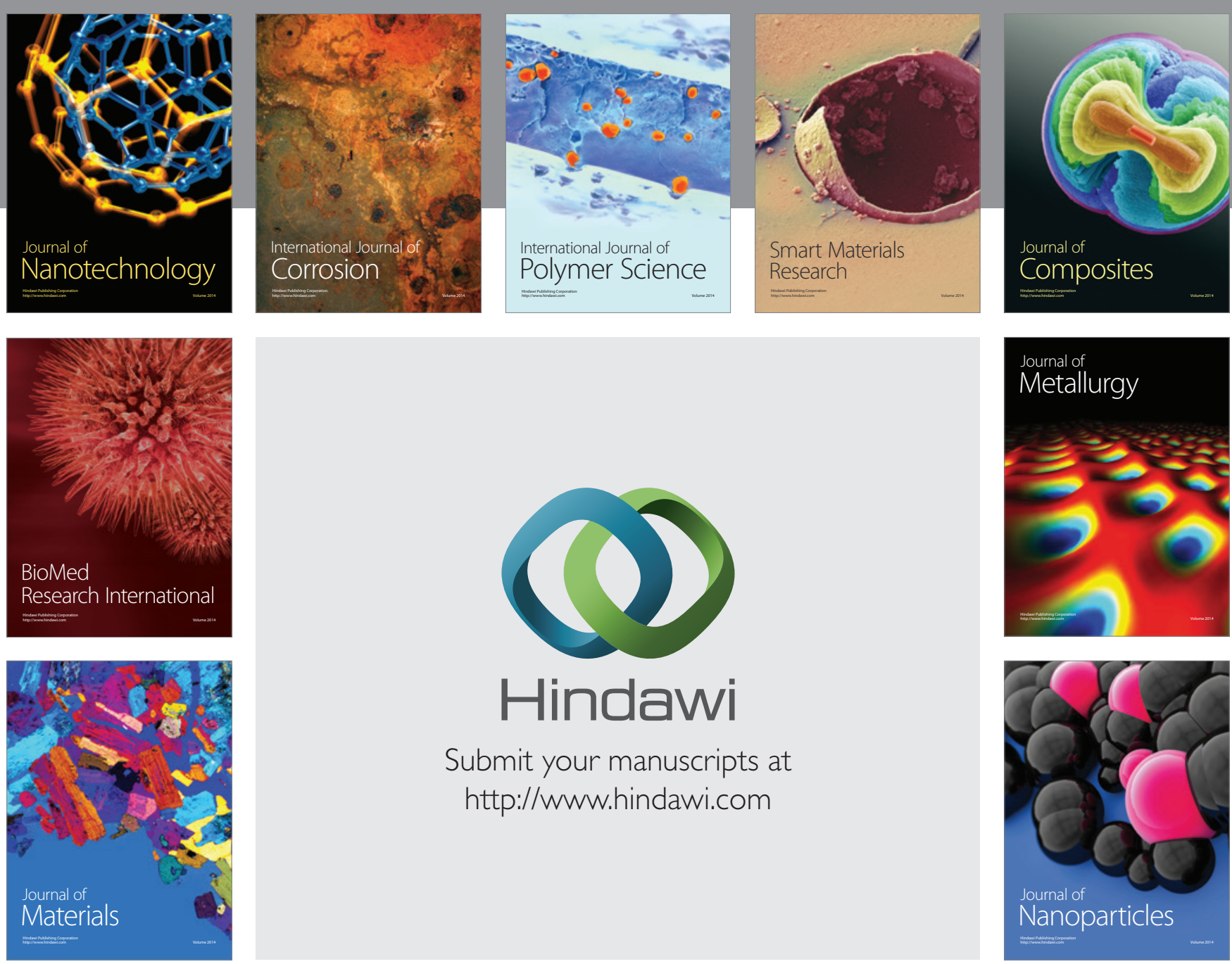

Submit your manuscripts at http://www.hindawi.com
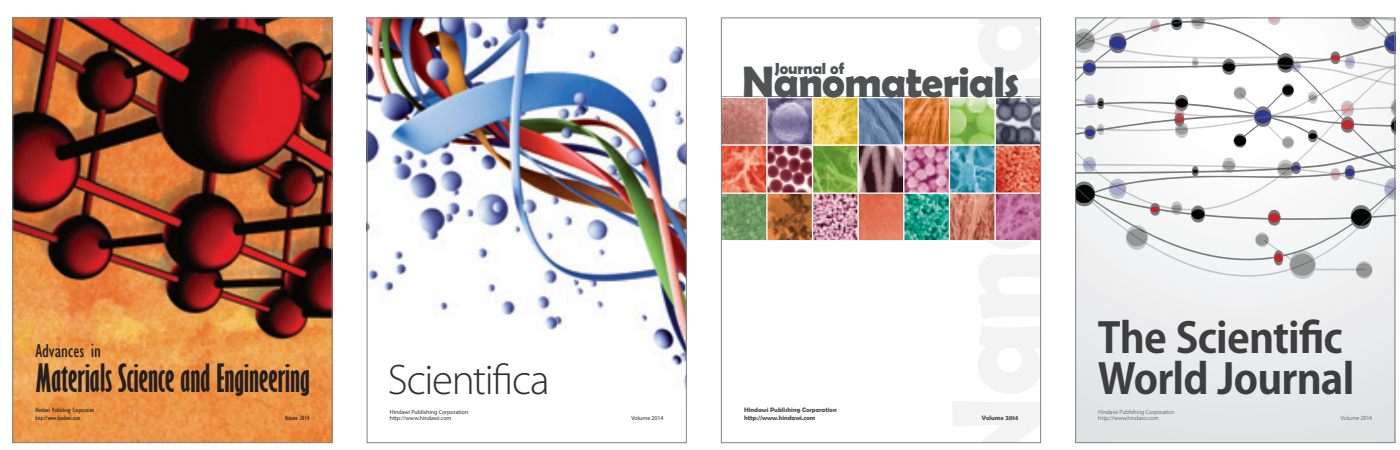

\section{The Scientific World Journal}
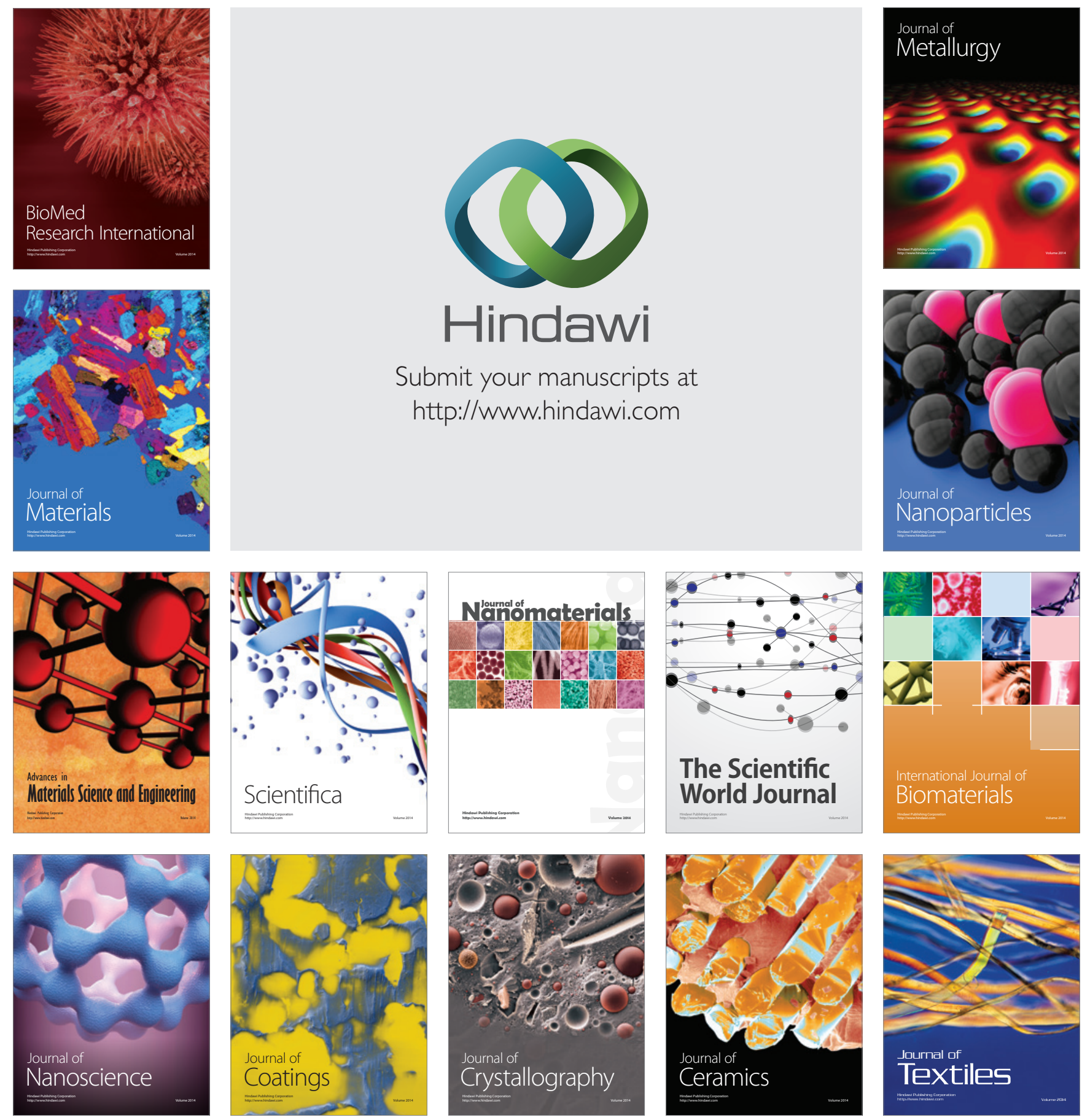